\title{
Contributos para o Estudo das Propriedades Psicométricas do BREQ-3
}

\author{
Contributions to the Study of the Psychometric Properties of BREQ-3
}

\author{
Isabel Simões Dias $^{1}$, Maria Odília Abreu² e Cláudia Figueiredo ${ }^{3}$
}

\begin{abstract}
Resumo
O presente estudo visa estudar as propriedades psicométricas da versão portuguesa do Behavioral Regulation in Exercise Questionnaire - 3 (BREQ-3). Este instrumento pretende avaliar a regulação comportamental para o exercício físico, baseando-se na teoria da autodeterminação de Deci e Ryan (1985). Um total de 996 participantes (823 mulheres, 82.6\%), estudantes, docentes e funcionários de uma Instituição do Ensino Superior Politécnico português, com idades compreendidas entre os 17 e os 68 anos $(\mathrm{M}=23.44$; $\mathrm{DP}=8.01)$, participou neste estudo. $\mathrm{O}$ modelo subjacente à construção do instrumento foi testado e obteve suporte empírico, sendo composto por 6 fatores que refletem constructos desde a amotivação até à regulação intrínseca e apresentou um bom ajustamento global (CFI=.983; TLI=.980; RMSEA=.058 CI90 [.050-.062]; $p=.040$ ). Os coeficientes alfa de Cronbach situaram-se entre .643 e .920 , revelando níveis adequados de fiabilidade. Embora sejam necessários estudos adicionais, o questionário revela indicadores que sustentam a sua qualidade para utilização na avaliação do tipo de motivação para a prática desportiva em adultos portugueses.
\end{abstract}

Palavras-chave: motivação, autodeterminação, exercício físico, propriedades psicométricas

\begin{abstract}
This study aims at analysing the psychometric properties of the Portuguese version of the Behavioral Regulation in Exercise Questionnaire (BREQ- 3). This instrument intends to assess the behavioural regulation concerning physical exercise, based on Deci and Ryan's self-determination theory. A total of 996 participants ( 823 females, $82.6 \%)$ students, teachers and staff of a Portuguese's polytechnic higher education institution, with ages ranging from 17 to 68 years $(M=23,44 ; \mathrm{DP}=8,1)$, participated in this study. The model underlying the instrument construction was tested and obtained empirical support, being composed by 6 factors, reflecting constructs from a motivation till intrinsic motivation, showed good global fit (CFI=.983; $\mathrm{TLI}=.980 ; \mathrm{RMSEA}=.058$, CI90 [.050-.062]; $p=.040$ ). Cronbach's alpha coefficients ranged from .643 to .920 showing adequate levels of reliability. Although additional studies are needed, the questionnaire shows indicators that support its quality for the assessment of the type of motivation to sports practice in Portuguese adults.
\end{abstract}

Keywords: motivation, self-determination, physical exercise, psychometric properties

\footnotetext{
${ }^{1} \mathrm{PhD}$ em Psicologia. Professora Adjunta, Escola Superior de Educação e Ciências Sociais/Instituto Politécnico de Leiria. Campus 1- Rua Dr. João Soares, Apartado 4045, 2411-901 Leiria, Portugal. Tel.: +351244829400. E-mail: isabel.dias@ipleiria.pt

${ }^{2}$ Mestre em Psicologia. Assistente Convidada, Escola Superior de Educação e Ciências Sociais/Instituto Politécnico de Leiria. Campus 1Rua Dr. João Soares, Apartado 4045, 2411-901 Leiria, Portugal. Tel.: +351244829400. E-mail: odilia.abreu@ipleiria.pt

${ }^{3} \mathrm{PhD}$ em Educação. Investigadora, GOVCOPP, Universidade de Aveiro. Campus Universitário de Santiago 3810-193 Aveiro, Portugal. Tel:+351919671564. E-mail: claudiafigueiredo@ua.pt

Revista Iberoamericana de Diagnóstico y Evaluación - e Avaliação Psicológica. RIDEP · №57 · Vol.4 · 53-63 · 2020

ISSN: 1135-3848 print /2183-6051online
} 


\section{Introdução}

Apesar de se reconhecerem os benefícios da prática regular de exercício físico para a saúde, a maior parte da população permanece reticente em envolver-se de forma regular em atividades físicas (Abreu \& Dias, 2016; Puigarnau, Camerino, Castañer, \& Hileno 2017; Veloso \& Loureiro, 2017). Este dado é particularmente preocupante na população portuguesa conforme mostram os dados do relatório da Comissão Europeia (2018), no qual se revela que a prática de exercício físico em Portugal é inferior à média europeia.

Uma explicação para a prática/não prática da atividade física e do exercício físico poder-se-á apoiar na teoria da autodeterminação de Deci e Ryan $(1985,1991,2008)$ que oferece um racional “(...) para a compreensão da sustentação dos comportamentos a longo prazo e princípios-guia para a prática" (Silva, Barata \& Teixeira, 2013, p. 36). Esta teoria conceptualiza as condições sociais que facilitam ou dificultam o sucesso humano, assumindo o ser humano como intrinsecamente curioso, fisicamente ativo e profundamente social (Abreu \& Dias, 2017; Ryan \& Deci, 2017).

Sendo uma macro-teoria da personalidade (Deci \& Ryan, 2008a) e do comportamento motivado, a teoria da autodeterminação foca a sua análise nas diferentes formas de motivação que são conceptualizadas num continuum de regulação comportamental que vai desde a regulação controlada (não-autónoma) até à autonomia (Gómez-Lopez, Granero-Gallego, BaenaExtremera, \& Abraldes, 2014; Teixeira, Carraca, Markland, Silva, \& Ryan, 2012). Ao estudar a motivação intrínseca e os processos de internalização (os seus determinantes e consequências), os autores descobriram a existência de condições universais para que cada forma de motivação possa surgir (Ryan, Huta, \& Deci, 2008). Sustentando que a satisfação de necessidades psicológicas básicas (autonomia, competência e desenvolvimento de relações sociais) e que o aumento da motivação autodeterminada medeiam o comportamento motivado (Deci \& Ryan, 2008; Serrano, Catalán, Remacha, \& Garcia-González, 2018), esta teoria defende que todos os comportamentos podem ser regulados por motivação controlada e autónoma. Assim, até chegar à motivação intrínseca (regulação autónoma), os sujeitos podem regular os seus comportamentos num continuum que oscila entre estilos regulatórios externos, introjetados ou interiorizados, identificados e integrados (Deci \& Ryan, 1985, 2008; Calmeira \& Matos, 2004), categorias discretas da motivação extrínseca. Este continuum de tipos de regulação (amotivação - motivação não regulada; motivação extrínseca - motivação regulada externamente e motivação intrínseca - motivação regulada intrinsecamente) varia ao longo da dimensão da autonomia (Teixeira et al., 2012; Wilson, Rodgers, Loitz, \& Scime, 2006) percorrendo um trajeto que vai passando da externalização (dependência do exterior) para internalização (autodeterminação) (ver Quadro 1).

Os processos de regulação são essenciais no estudo dos comportamentos promotores de saúde, nos quais se insere a prática de atividade física e de exercício físico (Teixeira, 2015). Neste sentido, existirá uma relação positiva entre formas de motivação autónoma e prática do exercício físico. A persistência nesta prática ocorrerá se os indivíduos estiverem intrinsecamente motivados (Cid, Moutão, Leitão, \& Alves, 2012), se tiverem mecanismos de tomada de decisão que conduzem à adoção de atitudes favoráveis face a essa mesma prática (Puigarnau et al., 2017), se tiverem consciência dos benefícios dessa mesma prática (Deci \& Ryan, 2008). Conforme Teixeira e colaboradores (2012), a regulação intrínseca será preditora da adesão ao exercício físico a longo prazo e a regulação identificada poderá predizer o início da prática do exercício físico. Assim, a regulação autodeterminada induzirá a maiores índices de envolvimento em atividades de exercício físico e terá um impacto positivo na perceção de satisfação com a vida.

Com o objetivo de aceder à avaliação da regulação comportamental em contexto de exercício físico, surge o Behavioral Regulation in Exercise Questionnaire (BREQ), da autoria de Mullan, Markland e Ingledew (1997) e baseado na teoria da autodeterminação de Deci e Ryan (1985, 1991). Este instrumento pretende avaliar o tipo de regulação utilizada na motivação extrínseca (regulação externa, introjetada, identificada) e a regulação usada na motivação intrínseca (regulação intrínseca), bem como a amotivação. Inicialmente composta por 5 subescalas, esta 
medida foi testada numa amostra de 298 participantes, mas a Análise Fatorial Confirmatória só permitiu confirmar 4 subescalas tendo sido retirada a subescala da amotivação (Mullan, Makland, \& Ingledew, 1997). Uma revisão realizada em 2004 por Markland e Tobin recupera a subescala da amotivação, considerando a necessidade do instrumento poder aceder à “ (...) ausência de intencionalidade de um sujeito de se envolver num comportamento (...)" (Markland \&Tobin, 2004, p. 191). Esta revisão resulta, então, no BREQ-2, instrumento que contém 5 subescalas que pretendem avaliar a regulação externa, a regulação introjetada, a regulação identificada, a regulação intrínseca e a amotivação. Porém, à luz da Teoria da Autodeterminação, tanto o BREQ como o BREQ-2 não contêm itens que avaliem a regulação integrada, sendo esta uma das formas de autorregulação que se situa na zona fronteira entre a motivação extrínseca e a motivação intrínseca. Esta questão é resolvida mais tarde, em 2006, quando Wilson, Rodgers, Loitz e Scime salientando a importância da regulação integrada como a forma mais autónoma de regulação dentro da motivação extrínseca e, desta forma, mais próxima da motivação intrínseca, validam 4 itens que pretendem aceder à regulação integrada. Estes 4 itens compõem a subescala da regulação integrada.

O BREQ-3 resulta, então, da justaposição do BREQ-2 e dos itens desenvolvidos por Wilson, Rodgers, Loitz e Scime em 2006 (Costa et al., 2017; Cid et al., 2018). Nesta versão o instrumento pretende medir as diferentes formas de regulação desde amotivação, regulação externa, introjetada, identificada, integrada (motivação extrínseca) e regulação intrínseca (motivação intrínseca) percorrendo todo o contínuo apresentado por Deci e Ryan na Teoria da autodeterminação $(1985,1991)$.

Nas suas diversas versões, este instrumento tem sido traduzido para diferentes línguas e adaptado para diferentes países (e.g.: Cid et al., 2018; Guedes \& Sofiati, 2015; Moreno, Cervelló, \& Martinez, 2007; Moustaka, Vlachopoulos, Vazou, Kaperoni, \& Markland, 2010) e populações distintas (e.g.: Costa et al., 2017; Sicilia, Sáenz-Alvarez, González-Cutre, \& Ferriz, 2014).

No caso de Portugal, Cid e colaboradores (2018) apresentaram um estudo para a validação do BREQ-3 com 24 itens: 19 itens do BREQ-2 (Markland \& Tobin, 2004), os 4 itens que compõem a subescala da regulação integrada propostos por Wilson e colaboradores (2006) e 1 novo item na subescala da regulação introjetada que, segundo os autores, resolve as limitações do BREQ-2 relacionadas com o item 17 (Cid et al., 2012). No trabalho que desenvolveram, recorreram a duas amostras independentes de praticantes de diferentes tipos de exercício físico em contexto de ginásio ( $\mathrm{N}=448 ; \mathrm{N}=374)$. Recorrendo a uma Análise Fatorial Confirmatória, validaram o instrumento com 6 fatores e 18 itens, tendo excluído 1 item por fator porque o instrumento como inicialmente previsto (com 24 itens e 6 fatores) "(...) não exibia propriedades psicométricas aceitáveis" (Cid et al., 2018, p. 8).

Ainda no contexto português, Costa e colaboradores (2017) apresentam um trabalho com o objetivo de validar o BREQ-3 numa população específica (pacientes com diagnóstico de esquizofrenia, $\mathrm{N}=118$ ) encontrando resultados bastante diferentes dos anteriormente enunciados. Neste trabalho, os resultados sugerem que a versão do BREQ-3 é um instrumento apropriado para medir a motivação controlada e autónoma em sujeitos com diagnóstico de esquizofrenia. "No entanto, algumas inconsistências foram identificadas nas análises da estrutura do BREQ-3 que podem dificultar a distinção clara entre as diferentes subescalas ao longo do continuum da motivação" (Costa et al., 2017, p. 5).

No contexto internacional de língua portuguesa, o estudo de Guedes e Sofiati (2015) com 1041 participantes recrutados em clubes de fitness da cidade de Londrina, estado de Paraná, no Brasil, constatou a total equivalência ao modelo original do BREQ-3, na sua tradução para português do Brasil. Assim, o teste ajusta-se ao modelo teórico com 23 itens organizados em 6 subescalas. Contudo, na versão brasileira não está incluído um item que consta da versão portuguesa (cf. Cid et al, 2018).

O presente estudo tem como objetivo explorar as propriedades psicométricas do BREQ-3 numa população de adultos portugueses (alunos, docentes e não docentes de uma instituição de ensino superior). A estrutura fatorial foi testada recorrendo a uma Análise Fatorial Confirmatória, 
fazendo uso de um estimador robusto que tem como pressuposto que os itens se expressam numa escala ordinal (como por definição podemos considerar as escalas de Likert; Kline, 2011; Muthén \& Muthén, 2001).

\section{Método}

\section{Participantes}

Os dados foram recolhidos através de uma amostra de conveniência num estabelecimento de ensino superior politécnico português. A totalidade dos participantes $(n=996)$ tem idades compreendidas entre os 17 e os 68 anos $(\mathrm{M}=23.44 ; \mathrm{DP}=8.01)$, e a grande maioria é do género feminino $(\mathrm{n}=823 ; 82.6 \%)$.

Os sujeitos afirmaram ter tido prática de exercício físico na última semana, no último ano, nos últimos cinco anos e nos últimos vinte anos acima de $40 \%$. No que refere à prática de exercício físico na última semana, $44 \%$ dos participantes $(n=438)$ referem ter praticado exercício físico, dos quais $20.5 \%(\mathrm{n}=204)$ pratica um vez ou menos por semana e 9.6\% ( $\mathrm{n}=96)$ pratica 2 vezes por semana. No último ano, $84.4 \%$ $(\mathrm{n}=841)$ dos sujeitos refere ter praticado exercício físico, dos quais $43.7 \%(n=435)$ pratica uma vez ou menos por semana e $19.3 \%(n=192)$ pratica 2 vezes por semana. Nos últimos cinco anos, $92.4 \%$ $(n=920)$ dos sujeitos refere ter praticado exercício físico, dos quais $47.0 \%(\mathrm{n}=468)$ pratica uma vez ou menos por semana e $23.4 \%(n=223)$ pratica 2 vezes por semana. Por fim, nos últimos vinte anos, 69.0\% ( $\mathrm{n}=687)$ dos sujeitos refere ter praticado exercício físico, dos quais $32.4 \%$ $(\mathrm{n}=323)$ pratica uma vez ou menos por semana e $17.5 \%(\mathrm{n}=174)$ pratica 2 vezes por semana.

\section{Instrumentos}

Contextualização da prática do exercício físico (Abreu \& Dias, 2015): este instrumento procura aceder aos dados sociodemográficos dos participantes bem como à informação acerca da prática do exercício físico (modalidades, frequência, duração) na última semana, no último ano, nos últimos cinco anos e nos últimos vinte anos.

Behavioral Regulation in Exercise Questionnaire (BREQ-3): O BREQ é usado para avaliar níveis de comportamento autodeterminado na prática do exercício físico. A versão portuguesa do BREQ-3 resulta dos estudos prévios realizados com o BREQ-2 da autoria de Palmeira e Teixeira (2006), de Palmeira, Teixeira, Silva e Markland (2007) e de Cid, Moutão, Leitão e Alves (2012). O BREQ-3 (Markland \& Tobin, 2004; Wilson et al., 2006), traduzido e adaptado para a população portuguesa por Cid e colaboradores (2018), tem 24 itens respondidos numa escala tipo Likert de 5 pontos, que varia entre "não é verdade para mim" (0) até "muitas vezes é verdade para mim" (4). Estes 24 itens organizam-se em 6 subescalas: amotivação, regulação externa, introjetada, identificada, integrada (motivação extrínseca) e regulação intrínseca (motivação intrínseca).

\section{Procedimento de recolha de dados}

A administração do BREQ-3 fez parte integrante de um protocolo de investigação num estudo mais amplo que visava estudar um conjunto alargado de variáveis (Abreu \& Dias, 2017). Após autorização da direção da instituição de ensino superior para a utilização do protocolo, o estudo foi apresentado aos participantes e estes foram informados acerca dos seus objetivos e da natureza voluntária e anónima do mesmo. O procedimento de recolha de dados assumiu duas formas distintas: resposta em contexto de sala de aula (em grupo), com o investigador presente, e resposta em contexto individual (sem presença do investigador) tendo sido entregue o protocolo ao participante. Os dados foram recolhidos num tempo aproximado de 15 minutos.

\section{Procedimento estatístico}

No tratamento estatístico dos dados recorreuse ao software MPlus 8 (Muthén \& Muthén, 2001) para os procedimentos de Análise Fatorial Confirmatória e ao SPSS 23 para a análise de fiabilidade.

Considerando a proposta teórica subjacente ao BREQ3 procedeu-se a uma Análise Fatorial Confirmatória (Byrne, 2012), recorrendo ao estimador WSLMSV (Weighted least square parameter using a diagonal weight matrix with robust errors and means). Esta opção pressupõe que um conjunto de variáveis manifestas ou indicadores ordinais marcam variáveis latentes numéricas (Muthén \& Muthén, 2001), como é o 
caso do presente instrumento com itens em escala de Likert de 5 pontos. Este estimador considerado robusto não implica pressupostos paramétricos de distribuição das variáveis, nomeadamente normalidade das distribuições. Uma vez que estamos perante um teste empírico à estrutura teórica conceptualizada para o instrumento em análise, será usada a designação "modelo" ao considerar o modelo estatístico que se encontra em análise.

$\mathrm{Na}$ avaliação da qualidade do ajustamento global ao modelo, recorreu-se à avaliação dos valores do teste de qui-quadrado. Contudo, sendo o resultado desta prova estatística sensível à dimensão da amostra e à complexidade do modelo, observou-se conjuntamente os seguintes índices de ajustamento global: CFI (Comparative Fit Index), TLI (Tucker and Lewis Index) e RMSEA (Root Mean Square Error of Approximation). A escolha destes indicadores é recomendada por se revelarem adequados dentro de um conjunto bastante extenso de indicadores que se encontram disponíveis e quando considerados conjuntamente (Hair, Black, Babin, Anderson, Tatham, 2006; Schermelleh-Engel, Moosbrugger, \& Müller, 2003). Considerou-se assim como pontos de corte para os referidos indicadores: maior que 90 para o CFI e TLI e menor que .07 para o RMSEA (Brown, 2006; Schermelleh-Engel et al., 2003).

$\mathrm{Na}$ análise do ajustamento local do modelo, ou seja, das estimativas de saturações fatoriais estandardizadas dos itens e dos valores de $R^{2}$ (contributo da variância de cada item para $\mathrm{o}$ modelo imposto), pretende-se perceber se os parâmetros estimados de forma ótima são interpretáveis considerando o que se conceptualiza acerca do constructo e da relação entre as suas dimensões. Assim, espera-se que as saturações fatoriais sejam estatisticamente significativas e superiores a .45 e que os valores de $\mathrm{R}^{2}$ evidenciem uma importante variância explicada por cada item para o fator que define (Brown, 2006; Hair et al., 2006; Tabachnick \& Fidell, 2007).

\section{Resultados}

O BREQ-3 é conceptualmente composto por 6 fatores correlacionados entre si que são definidos a partir de um total de 24 variáveis observadas (itens), 4 por variável latente (fator).

Considerando que esta estrutura conceptual tem uma relevante importância para a intervenção psicológica na regulação comportamental para o exercício físico, realizou-se o teste inicial de ajustamento ao modelo empírico subjacente à construção do BREQ-3 proposta pelos autores. Os resultados deste teste revelaram valores de ajustamento aceitáveis, mas onde se observava, contudo, multicolinearidade muito elevada de uma variável manifesta (item 21) relativamente a várias outras, quer pertencentes ao mesmo fator, quer a outros. Nesse sentido, para melhorar a qualidade da medida subjacente ao modelo em teste, optou-se por eliminar da análise o referido item (BREQ21). O item 21 pertence à subescala Regulação Introjetada (IJ - Factor 3) na versão portuguesa (Cid et al., 2018). A sua formulação "Sinto-me ansioso(a) se não fizer exercício regularmente", pode ser interpretada de diferentes formas, ambiguidade que pode justificar que esteja correlacionado com vários outros itens de outras subescalas. Esta afirmação pode ser interpretada, por exemplo, como "ansiedade" associada a pressões externas (Regulação Externa); como ansiedade associada a pressões internas (Regulação Introjetada); ou como ansiedade associada ao não cumprimento de valores internos (Regulação Identificada).

O modelo colocado em teste com a nova configuração (cf. Figura 1), apresenta um teste formal de hipótese estatisticamente significativo $\left(\chi^{2}(\mathrm{~N}=996,215)=931.696 ; p<.001\right)$. Considerando a improbabilidade de se obter um valor sem significância estatística foram considerados adicionalmente os seguintes indicadores de ajustamento global: $\quad$ CFI $=.983 ; \quad$ TLI $=.980$; RMSEA $=.058$ CI90 [.054-.062]; $p<.001$.

O CFI e o TLI estão acima do ponto de corte recomendado (>.95) enquanto o RMSEA abaixo do ponto de referência (<.07; Brown, 2006; Schermelleh-Engel et al, 2003).

A análise conjunta do teste estatístico e dos restantes indicadores de ajustamento global permite afirmar pela aceitação da estrutura das variáveis como sendo ajustada ao modelo teórico original.

$\mathrm{Na}$ análise das estimativas pontuais do modelo verifica-se que todas as saturações fatoriais são 


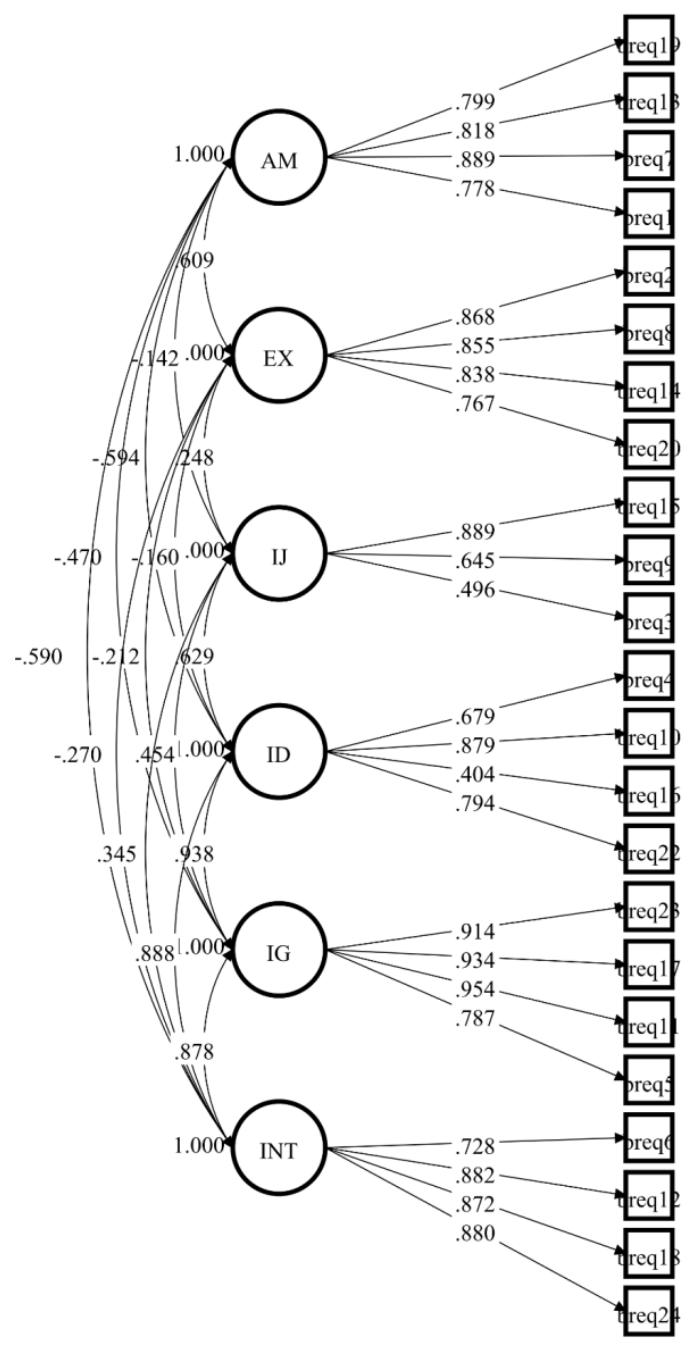

Figura 1. Especificação pictográfica do modelo em teste; estimativas estandardizadas ( $\mathrm{n}=996)$ Nota. Amotivação - AM; Regulação Externa - EX; Regulação Introjetada - IJ; Regulação Identificada - ID; Regulação Integrada IG; Regulação Intrínseca - INT

estatisticamente significativas e superiores a .45 , considerado um valor razoável a bom por Tabachnick e Fidell (2007). Todas as variáveis observadas são explicadas pelo modelo a pelo menos $20 \%$. A variância explicada por indicador observado varia entre .238 (BREQ16) e .912 (BREQ11).

O primeiro fator (Amotivação - AM) possui 4 variáveis observadas com saturações fatoriais a variar entre .764 (BREQ1) e .939 (BREQ7). O segundo fator (Regulação Externa - EX), 4 variáveis observadas com saturações entre .747 (BREQ20) e .897 (BREQ2). O terceiro fator (Regulação Introjetada - $\quad$ IJ), 3 variáveis observadas com saturações fatoriais entre .505 (BREQ3) e .895 (BREQ15). O quarto fator (Regulação Identificada - ID), com 4 variáveis observadas cujas saturações se situam ente .487
(BREQ16). O quinto fator (Regulação Integrada IG) é composto por 4 variáveis observadas com saturações fatoriais a variar entre .765 (BREQ5) e .955 (BREQ11). O sexto, e último fator, Regulação Intrínseca - IM, inclui 4 indicadores observados com saturações fatoriais entre .687 (BREQ6) e .888 (BREQ18) (Quadro 1).

As correlações item-total corrigidas (r) bem como os coeficientes alfa de Cronbach permitem confirmar a boa fiabilidade de todas as seis dimensões do instrumento. $\mathrm{O}$ indicador de consistência interna apresentou valores entre .643 (IJ) e .920 (ID), cinco dos seis superiores a .70, revelando níveis adequados de consistência interna, sobretudo se tomarmos em consideração o reduzido número de itens por dimensão (DeVellis, 2012; Quadro 1).

Relativamente aos níveis de associação entre 
Quadro 1. Coeficientes estandardizados, coeficientes de determinação $\left(R^{2}\right)$ e correlação item total corrigido ( $r$ ) do modelo final da BREQ-3 $(n=996)$

\begin{tabular}{|c|c|c|c|c|c|c|c|c|c|}
\hline & \multirow{2}{*}{$\begin{array}{c}\text { Item } \\
\text { Conteúdo }\end{array}$} & \multicolumn{6}{|c|}{ Fatores } & \multirow[t]{2}{*}{$R^{2}$} & \multirow[t]{2}{*}{$r$} \\
\hline & & $\mathrm{AM}$ & EX & IJ & ID & IG & INT & & \\
\hline BREQ1 & & .764 & & & & & & .584 & .464 \\
\hline BREQ7 & & .939 & & & & & & .881 & .641 \\
\hline BREQ13 & & .821 & & & & & & .674 & .534 \\
\hline BREQ19 & & .794 & & & & & & .630 & .467 \\
\hline BREQ2 & & & .897 & & & & & .805 & .639 \\
\hline BREQ8 & & & .855 & & & & & .731 & .687 \\
\hline BREQ14 & & & .838 & & & & & .703 & .527 \\
\hline BREQ20 & & & .747 & & & & & .558 & .525 \\
\hline BREQ3 & & & & .505 & & & & .255 & .452 \\
\hline BREQ9 & & & & .635 & & & & .403 & .419 \\
\hline BREQ15 & & & & .895 & & & & .802 & .499 \\
\hline BREQ4 & & & & & .700 & & & .489 & .482 \\
\hline BREQ10 & & & & & .878 & & & .771 & .630 \\
\hline BREQ16 & & & & & .487 & & & .238 & .368 \\
\hline BREQ22 & & & & & .791 & & & .626 & .534 \\
\hline BREQ5 & & & & & & .765 & & .585 & .715 \\
\hline BREQ11 & & & & & & .955 & & .912 & .874 \\
\hline BREQ17 & & & & & & .938 & & .880 & .868 \\
\hline BREQ23 & & & & & & .913 & & .834 & .807 \\
\hline BREQ6 & & & & & & & .687 & .472 & .642 \\
\hline BREQ12 & & & & & & & .886 & .785 & .769 \\
\hline BREQ18 & & & & & & & .888 & .789 & .764 \\
\hline BREQ24 & & & & & & & .886 & .785 & .722 \\
\hline Alfa de Cronbach & & .738 & .782 & .643 & .711 & .920 & .868 & & \\
\hline
\end{tabular}

Nota. Amotivação-AM; Regulação Externa-EX; Regulação Introjetada-IJ; Regulação Identificada-ID; Regulação Integrada-IG; Regulação Intrínseca-INT

Quadro 2. Médias, desvios-padrão e matriz de correlação entre os fatores do modelo

\begin{tabular}{lcccccccc}
\hline \multicolumn{1}{r}{$\mathrm{r}$} & $\mathrm{M}$ & $\mathrm{DP}$ & $\mathrm{AM}$ & $\mathrm{EX}$ & IJ & ID & IG & INT \\
\hline AM & 1.28 & 0.56 & 1.000 & & & & & \\
EX & 1.36 & 0.60 & $.479^{*}$ & 1.000 & & & & \\
IJ & 2.47 & 0.97 & $-.237^{*}$ & $.225^{*}$ & 1.000 & & & \\
ID & 3.68 & 0.83 & $-.593^{*}$ & -.086 & $.629^{*}$ & 1.000 & & \\
IG & 3.14 & 1.25 & $-480^{*}$ & $-.247^{*}$ & $.446^{*}$ & $.939^{*}$ & 1.000 & \\
INT & 3.73 & 1.02 & $-.579^{*}$ & $-.247^{*}$ & $.390^{*}$ & $.883^{*}$ & $.877^{*}$ & 1.000 \\
\hline
\end{tabular}

Nota. *p<.001; Amotivação-AM; Regulação Externa-EX; Regulação Introjetada-IJ; Regulação Identificada-ID; Regulação Integrada-IG; Regulação Intrínseca-INT

os fatores verifica-se que todos os coeficientes são estatisticamente significativos com exceção da correlação entre EX e ID. Os valores da correlação entre os restantes fatores variam entre 225 (EX com o IJ) e .939 (ID com o IG) (Quadro 2).

A análise descritiva das pontuações dos participantes nas diferentes dimensões permite observar que a média mais elevada corresponde à Regulação Intrínseca para a prática desportiva $(\mathrm{M}=3.73 ; \quad \mathrm{DP}=1.02)$, seguida de Regulação Identificada $(\mathrm{M}=3.68 ; \quad \mathrm{DP}=0.83)$. Amotivação $(\mathrm{M}=1.28$; $\mathrm{DP}=0.56)$ e Regulação Externa $(\mathrm{M}=1.36$; $\mathrm{DP}=0.60)$ são as dimensões que revelam uma pontuação mais baixa (Quadro 2).

\section{Discussão}

Este trabalho, procurando contribuir para o estudo das qualidades psicométricas da versão portuguesa do BREQ-3, revela uma estrutura fatorial muito aproximada ao modelo conceptual no qual o instrumento se baseia (Deci \& Ryan, 1985, 1991, 2008; Wilson et al., 2006; Markland \& Tobin, 2004). O modelo estatístico testado confirmou uma estrutura composta por 6 fatores, considerando os itens definidos em cada uma destas variáveis latentes, apenas com a necessidade de exclusão de um item dos 24 iniciais (BREQ21), na dimensão IJ. 
O modelo empírico testado apresentava 6 fatores compostos por 4 itens cada um tal como o inicialmente previsto na construção do BREQ-3 prevê (Cid, Monteiro, Moutão, Teques, Silva, Teixeira, \& Palmeira, 2015; Teixeira, Silva, \& Palmeira, 2018). No entanto, o item 21 foi retirado por os resultados estatísticos darem a indicação de este não se revelar específico do fator a que supostamente pertencia (IJ).

Estes resultados distinguem-se dos encontrados por Cid e colaboradores (2018) para a população portuguesa. O estudo realizado por estes autores concluiu pela necessidade de retirar um item em cada subescala, mantendo os seis fatores mas com apenas 18 itens. Saliente-se que as amostras destes dois trabalhos não são comparáveis, uma vez que não preenchem os mesmos critérios. No presente estudo, optou-se por uma amostra de conveniência e no estudo de Cid e colaboradores (2018), a amostra preenche um conjunto de critérios previamente definidos e que passam pela seleção de sujeitos após uma sessão de prática de exercício físico. No presente estudo os dados não foram recolhidos em contexto de exercício físico (após a prática de exercício físico), embora o protocolo de investigação fosse acompanhado de um instrumento sociodemográfico que permite ao participante identificar a prática de exercício físico. No estudo de Cid e colaboradores (2018) os dados foram recolhidos após a prática de atividades de ginásio o que garante a existência efetiva da prática, mas não apresenta informação relativa aos sujeitos que têm práticas de atividade física mais moderadas ou de forma menos estruturada.

A nível internacional, num estudo realizado por Guedes e Sofiati (2015) num país de língua portuguesa, com uma amostra de 1041 sujeitos adultos, frequentadores de um clube de fitness, a Análise Fatorial Confirmatória, confirmou a estrutura de 6 fatores originalmente proposta, à semelhança dos resultados encontrados no presente trabalho.

Os estudos de Cid colaboradores (2018) e Guedes e Sofiati (2015) utilizam um conjunto de participantes que responderam imediatamente após a prática de exercício físico estruturado. No presente estudo, foram incluídos todos os sujeitos, quer referissem praticar ou não exercício físico. Dos respondentes $44 \%$ afirmou ter praticado regularmente exercício físico na última semana. Embora esta amostra não represente a população portuguesa, esta percentagem está acima da média nacional, considerando os dados do Eurobarómetro 2017. De acordo com o Eurobarómetro, a percentagem de portugueses que praticou exercício ou desporto regularmente foi, em 2017, de 5\%. A mesma fonte refere que, em 2017, a prática de atividades físicas de intensidade moderada pela população portuguesa (e.g.: transportar objetos leves, jogar ténis em pares), realizada uma a três vezes por semana foi de $17 \%$ (27\% na Europa). Já a atividade física de intensidade vigorosa (e.g.: levantar objetos pesados, exercício físico aeróbio), realizada, entre uma a três vezes por semana foi de $13 \%$ (29\% na Europa). Estes indicadores mostram que a prática de exercício físico no nosso país é inferior à média europeia. Atividades como andar de bicicleta dançar ou fazer jardinagem são referidas somente por $5 \%$ da população portuguesa (Comissão Europeia, 2018).

$\mathrm{O}$ teste efetuado no presente estudo à estrutura dimensional do BREQ-3 recorreu a um modelo de equações estruturais considerando um estimador específico para dados recolhidos em escalas ordinais, tal como a escala de Likert usada pelo instrumento. Desta forma, será possível assumir excelentes qualidades psicométricas do BREQ-3 na população de ensino superior em concreto. As mesmas qualidades psicométricas podem ser esperadas numa população geral com níveis de literacia médio-altos.

A plausibilidade empírica do modelo conceptual subjacente à construção do BREQ3 permite suportar a intervenção de psicólogos e profissionais na regulação comportamental para o exercício físico, salvaguardando-se que podem existir outros modelos igualmente plausíveis subjacentes a este mesmo constructo.

Ao realizar o estudo das qualidades psicométrica do BREQ-3, foi possível observar que os participantes, relativamente à prática do exercício físico, se consideraram num continuum motivacional (Deci \& Ryan, 1985, 1991, 2008). Os participantes revelaram formas de regulação autónoma (regulação identificada, regulação integrada e regulação intrínseca). Estes dados possibilitam equacionar a hipótese de que serão sujeitos que persistem na prática (Cid, Moutão, 
Leitão, \& Alves, 2012), com maior probabilidade de aderir ao exercício físico a longo prazo (Teixeira et al., 2012) e/ou, com maior probabilidade de iniciarem uma prática de exercício físico (Teixeira, 2015).

Em futuros estudos, pretende-se comparar uma amostra de sujeitos praticantes de desporto federado (modalidades individuais vs coletivas) com sujeitos que praticam exercício regularmente (e.g.: modalidades de ginásio) e com sujeitos que referem nunca praticar exercício físico.

$\mathrm{O}$ presente estudo evidencia as excelentes qualidades psicométricas do BREQ-3 numa população do ensino superior politécnico nacional que afirmou ter uma prática de exercício físico superior à média da população portuguesa.

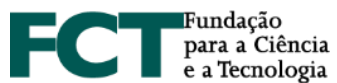

\section{UID/CED/04748/2020}

\section{Referências}

Abreu, M. O., \& Dias, I.S. (2016). Relação entre qualidade de vida e exercício físico em contexto de Ensino Superior. Revista da UIIPS, 4(2), p.216-229.

doi:https://doi.org/10.25746/ruiips.v4.i2.14433

Abreu, M. A., \& Dias, I.S. (2015). Qualidade de vida e exercício físico: Descrição de uma amostra na ESECS/IPL. Comunicação apresentada na Conferência Internacional Intervenção, Práticas e Contextos em Educação (IPCE 2015), Leiria, Portugal.

Abreu, M. O., \& Dias, I. S. (2017). Exercício físico, saúde mental e qualidade de vida na ESECS/IPL. Psicologia, Saúde \& Doenças, 18(2), 512-526. doi:10.15309/17psd180219

Brown, T. (2006). Confirmatory factor analysis for applied research. New York: Guilford Press.

Byrne, B. (2012). Structural equation modeling with Mplus: Basic concepts, applications and programming. New York: Routledge.

Calmeiro, L., \& Matos, M. (2004). Psicologia, Exercício e Saúde. Lisboa: Visão e Contextos.

Cid, L., Monteiro, D., Moutão, J., Teques, P., Silva, M., Teixeira, D. S., \& Palmeira, A. L.
(2015). Validação do behavioral regulation in exercise questionnaire numa amostra de praticantes de exercício portugueses com análise de invariância entre géneros. Comunicação apresentada nas XVII Jornadas da Sociedade Portuguesa de Psicologia do Desporto, Setúbal, Portugal.

Cid, L., Monteiro, D., Teixeira, D., Teques, P., Alves, S., Moutão, J., Silva, M., \& Palmeira, A. (2018). The behavioral regulation in exercise questionnaire (BREQ-3) Portugueseversion: evidence of reliability, validity and invariance across gender. Frontiers in Psychology, 9, 1-9. doi:10.3389/fpsyg.2018.01940

Cid, L., Moutão, J., Leitão, J., \& Alves, J. (2012). Behavioral regulation assessment in exercise: Exploring an autonomous and controlled motivation index. The Spanish Journal of Psychology, 15(3), 1520-1528. doi:10.5209/rev_SJOP.2012.v15.n3.39436

Costa, R., Prosbt, M., Bastos, T., Vilhena, E., Seabra, A., \& Corredeira, R. (2017). Behavioral regulation in Exercise Questionnaire in people with schizophrenia: construct validity of the Portuguese versions. Disability and Rehabilitation, 40(12), 25772584. doi:10.1080/09638288.2017.1342277

Deci, E., \& Ryan, R. (2008). Facilitating optimal motivation and psychological well-being across life's domains. Canadian Psychology, 49(1), 14-23. doi:10.1037/0708-5591.49.1.14

Deci, E., \& Ryan, R. (2008a). Self-Determination Theory: A Macrotheory of Human Motivation, Development, and Health. Canadian Psychology, 49(3), 182-185. doi:10.1037/a001280

Deci, E. L., \& Ryan, R. M. (1985). Intrinsic motivation and self-determination in human behavior. New York: Plenum.

Deci, E. L., \& Ryan, R. M. (1991). A motivational approach to self: Integration in personality. In R. Dienstbier (Ed.). Nebraska Symposium on Motivation Vol 38: Perspectives on Motivation (pp. 237-288). Lincoln: University of Nebraska Press.

DeVellis, R. (2012). Scale development: theory and applications (3rd ed.). Thousand Oaks: Sage. 
European Commission Directorate-General for Education, Youth, Sport and Culture (2018). Special Eurobarometer 472 - December 2017 "Sport and physical activity" Report. Brussels: European Comission. doi: 10.2766/483047

Gómez-Lopez, M., Granero-Gallego, A., BaenaExtremera, A., \& Abraldes, J. (2014). Análisis de los perfiles motivacionales y su relación con la importancia de la educación física en secundaria. Revista Iberoamericana de Diagnóstico y Evaluación - e Avaliação Psicológica, 38(2), 11-29.

Guedes, D., \& Sofiati, S. (2015). Translation and psychometric validation of the behavioral regulation in exercise questionnaire for use in Brazilian adults. Revista Brasileira de Atividade Física \& Saúde 20, 397-412. doi:10.12820/RBAFS.V.20N4P397

Hair, J., Black, W., Babin, B., Anderson, R., \& Tatham, R. (2006). Multivariate data analysis (6th ed.). New Jersey: Pearson Prentice Hall.

Kline, R. (2011). Principles and practice of structural equation modeling ( $3^{\text {rd }}$ ed.). New York: The Guilford Press.

Markland, D. A., \& Tobin, V. (2004). A modification to the behavioural regulation in exercise questionnaire to include an assessment of amotivation. Journal of Sport \& Exercise Psychology, 26, 191-196. doi:10.1123/jsep.26.2.191

Moreno, J. A., Cervelló, E. M., \& Martínez, A. (2007). Measuring self-determination motivation in a physical fitness setting: Validation of the Behavioral Regulation in Exercise Questionnaire-2 (BREQ-2) in a Spanish sample. The Journal of Sport Medicine and Physical Fitness, 47, 366-378.

Moustaka, F. C., Vlachopoulos, S. P., Vazou, S., Kaperoni, M., \& Markland, D. (2010). Initial validity evidence for the Behavioural Regulation in Exercise Questionnaire - 2 among Greek exercise participants. European Journal of Psychological Assessment, 26, 269-276. doi:10.1027/1015-5759/a000036

Mullan, E., Markland D., \& Ingledew, D. K. (1997). Motivation for exercise: Development of a measure of behavioral regulation. Journal of Sports Sciences, 15, 98-99. doi:10.1080/08870440701405704
Muthén, L., \& Muthén, B. (2001). Mplus user's guide: Statistical analysis with latent variables. Los Angeles: Muthén \& Muthén.

Palmeira, A. L., \& Teixeira, P. J. (2006). Adaptação preliminar do behavioural regulation in exercise questionnaire (BREQ2) para a língua portuguesa. Comunicação apresentada no V Congresso Luso-Espanhol de Psicologia do Desporto, Lisboa, Portugal.

Palmeira, A., Teixeira, P., Silva, M., \& Markland, D. (2007). Confirmatory factor analysis of the BREQ-2 - Portuguese version. Comunicação apresentada no 12th European Congress of Sport Psychology, Halkidiki, Grécia.

Puigarnau, S., Camerino, O., Castañer, M., \& Hileno, R. (2017). Self-determination in a pshysical exercise program to promote healthy habits in sedentary adults: A mixed methods approach, Psychological and Behavioral Science, 7(3), 001-008. doi:10.19080/PBSIJ.2017.07.555714

Ryan, R., Huta, V., \& Deci, E. (2008). Living well: A self-determination theory perspective on eudaimonia. Journal of Happiness Studies, 9, 139-170. doi:10. 1007/s10902-006-9023-4.

Ryan, R., \& Deci, E. (2017). Self-determination theory. Basic psychological needs in motivation, development and wellness. New York: The Guilford Press.

Schermelleh-Engel, K., Moosbrugger, H., \& Müller, H. (2003). Evaluating the fit of structural equation models: Tests of significance and descriptive goodness-of-fit measures. Methods of Psychological Research Online, 8(2), 23-74.

Serrano, J., Catalán, A., Remacha, M., \& GarciaGonzález, L. (2018). El "Lado Claro" y El "Lado Oscuro" de la Motivación en Educación Física: Efectos de una intervención en una unidad didáctica de atletismo. Revista Iberoamericana de Diagnóstico y Evaluación - e Avaliação Psicológica, 46(1), 93-107. doi:10.21865/PIDEP46.1.07

Sicilia, A., Sáenz-Alvarez, P., González-Cutre, D., \& Ferriz, R. (2014). Exercise motivation and social physique anxiety in adolescents. Psychologica Belgica, 54(1), 111-129, doi:10.5334/pb.ai

Silva, M., Barata, J., \& Teixeira, P. (2013). Exercício físico na diabetes: Missão 
impossível ou uma questão de motivação? Revista Portuguesa de Cardiología, 32(supl.I), 35-43.

Tabachnick, B., \& Fidell, L. (2007). Using multivariate statistics (5th ed.). USA: Pearson Education.

Teixeira, D., Silva, M., \& Palmeira, A. (2018). How does frustration make you feel? A motivational analysis in exercise context, Motivation and Emotion, 42(3), 419-428. doi:10.1007/s11031-018-9690-6.

Teixeira, P. (2015). A primer on self-regulation and health behavior. Arch Exerc Health Dis, 5(1-2), 326-337. doi:10.5628/aehd.v5i1-2.184.

Teixeira, P., Carraca, E., Markland, D., Silva, M., \& Ryan, R. (2012). Exercise, physical activity, and self-determination theory: A systematic review. International Journal of Behavioral Nutrition and Physical Activity, 978. https://doi.org/10.1186/1479-5868-9-78

Veloso, S., \& Loureiro, A. (2017). Exercise and nature: A relevant combination to health and well-being, Revista Iberoamericana de Psicología del Ejercício y el Desporte, 12(2), 313-319.

Wilson, P. M., Rodgers, W. M., Loitz, C. C., \& Scime, G. (2006). "It's who I am...really!" The importance of integrated regulation in exercise contexts. Journal of Biobehavioral Research, 11, 79-104. doi:10.1111/j.1751-9861.2006.tb00021.x 